\title{
A Strategy of Dynamic Replica Creation in Cloud Storage
}

\author{
Mengxing Huang, Xianglong Ye, Sanpeng Wei, Donghai Zhu \\ College of Information Science \& Technology \\ Hainan University \\ Haikou, China \\ huangmx09@gmail.com
}

\begin{abstract}
With the popularity of cloud storage, its reliability and access latency have become increasingly prominent. To solve this problem, a novel strategy of dynamic replica creation based on acceleration is presented for cloud storage by drawing lessons from the concept of acceleration. For reducing access latency, the strategy predicts the hot files in the way of acceleration based on accessing locality principle. Finally, the performances of the strategy of acceleration replicas creation and the strategy of best client replicas creation are simulated and analyzed. The simulation results show that the strategy of acceleration replicas creation predicted hot files in the next period of time more accurately than the strategy of best client replicas creation, reduced network latency more than the strategy of best clients and the effect was being more pronounced with accessing trends rising. The strategy of acceleration replicas creation is suitable for cloud storage which is for handling massive files. By this strategy, not only the reliability of cloud storage data is improved, but also access latency is reduced.
\end{abstract}

Keywords - Access latency, Hot files, Replicas management

\section{INTRODUCTION}

With the development of Internet technology, especially Web 2.0 application has been widely used, the information is not only deeply affect people's lives, but also the advent of the Internet information explosion. It is important that how to make huge amounts of data storage and management, and how to ensure the cost is low and does not lose data efficiency, reliability, fault tolerance and so on. Cloud storage is produced in such an environment, it not only in terms of price to cheap storage service, also in performance to provide a simpler and more effective way to store and manage mass data and information explosion, etc. Cloud storage is through the establishment of large data centers and large data centers deployed in a large number of low-cost PC, to provide people with low-cost, efficient, highly reliable, fault-tolerant services [1-3]. However, just because of the extensive use of the low-cost PC's, performance is not high, when a large number of users to access the same file, which is easy to access delay, or even the node crashes and other problems. To solve this problem, the cloud storage with replica technology improved data availability, reliability, high efficiency, high fault tolerance and so on.

But the introduction of replica technology also will bring a waste of resources, increase the cost of the system running, replica consistency problem.
Replica management is to manage the number and location of a copy, which has always been a hot issue of research. Replica management contains the static management and dynamic management. Static replica management is that the number and storage location of replica were decided when file created initially which did not change during system operation.

Dynamic replica management is to dynamically change the number and location of replica according to the real-time needs of the system. The key problem of the dynamic replica management is to create a replica dynamically which concern the creation time, the creation granularity, the number and the location. A good dynamic to create a copy of the strategy can greatly improve the efficiency of user access. In contrast, a poor performance of the dynamic replication strategy is not only unable to improve the system operation status, copy also increases the management cost.

Cloud storage is a new research field; the dynamic replication strategy is still rarely seen. In this paper, the focus is on early distributed file system replica creation strategy, combining with the characteristics of cloud storage, we design a dynamic replication strategy based on acceleration .It create a replica according to the file access history characteristics, so that users can access the nearest which brings the system to one of the best state, namely the replica number of minimum, access to the highest efficiency, network latency minimum.

\section{II . RELATED WORK}

Replica creation improved the cloud storage system reliability, fault tolerance, user access efficiency and reduce network latency, and avoided node failure or crash. According to the replicas will change along with the running of the system after were created, replica creation contains the static replica creation strategy and the dynamic replica creation strategy.

\section{A. Static Replica Creation Stragedy}

Due to its characteristics such as easy to implement, HDFS also adopt this policy by default [4]. HDFS placed way is to put the first replica in the local node, then put the second replica in another node which at local rack randomly. At last, put the third replica put into different nodes on the rack randomly. This approach reduces rack write traffic and improves write performance. Chance of rack failure is far less than the effectiveness of the machine. This way not only guarantees for the reliability and availability of data, but also reduces the read operation of the network aggregate bandwidth. However, 
the system does not take into account the node space utilization when creating a replica.

In view of the above questions, the literature [5] on HDFS replication strategy is improved .From the choice of replication nodes, in order to load balance as the goal, this strategy selects the optimal node according to node real-time dynamically. The improvement strategy in the literature takes into account the system's real-time condition when creating a replica. It can well adapt to changes in the system when system running and can effectively avoid the problem of load imbalance. However, this strategy creates a replica which does not change in the future operation of the system .This strategy has shortcomings when the users demand change a lot.

\section{B. Dynamic Replica Creation Stragedy}

This strategy is more flexible and replica is no longer static but will change with the change of the operation of the system which can be well adapted to the changes in the system.

Literature [6] describes the six kinds of replica creation strategy. The six strategies are: no replica strategy, the best client strategy, waterfall strategy, caching strategy, waterfall combining with caching strategy and rapid spread strategy. These strategies in most cases in other distributed environment can reduce the user access latency and save network resources. However, waterfall strategy applies only to hierarchical network. It cannot adapt to the complicated and changeable cloud storage system .Rapid spread strategy can be stored in a node in the system only for small size, small visits, individual files .This is obviously not suitable to solve massive data which access to a large amount of cloud storage system. Caching strategy and best client strategy create a replica only based on user visits. This strategy cannot response the problem of hot spot data in the next period and cannot be very good to find a file which needs to create a replica and create a replica for it.

There is a replica creation strategy based on economic models is presented [7]. This strategy uses file transfer time as a metric of auction price and selects nodes and creates a replica based on reverse auction protocol. This strategy has good performance, but it is based on the European data grid design. Creating and deleting a replica is most likely in low storage capacity of the node. This is not suitable for deployment in the cloud storage system on the cheap PC.

Later, Hassan [8] put forward their own dynamic replication strategy from reducing message traffic. Feng Guofu [9] proposed initiative replication strategy from the point of view of the P2P overlay network topology. Kyungbaek Kim [10] proposed a time-related replication strategy which is the use the node interaction information to prevent loss of replica. Xin Sun [11] put forward a dynamic replication strategy based on access costs for the purpose of reduced delays. They are designed for distributed environments and dynamic replication strategy. They cannot adapt well to the cloud storage system which can handle huge amounts of data and bear huge traffic.

In terms of cloud storage, some people also made a study on the dynamic replication strategy. Xu Jing [12] puts forward by introducing the market mechanism of price, considering the balance of the load balance and consistency and availability .But this strategy can not neither reduce the network latency nor provide user access efficiency.

\section{REPLICA CREATION MODULE IN CLOUD STORAGE}

Cloud storage is a service which is provided services to users through the establishment of one or more data centers. In order to provide efficient, reliable cloud storage services, cloud storage adopted replica strategy. Cloud storage is a technology which exists to solve the massive data, thousands or even more users to access the same file at the same time. So it should cerate multiple replicas when creation a file in order to ensure the reliability and efficiency of cloud storage, etc .For this problem, dynamic replication strategy and static replication strategy should be provided.

As shown in figure 1 , this is a cloud storage replica creation model .In this model which contains dynamic replica creation module and static replica creation module. When the cloud-client need request to create a file, it makes a request for the name node to create a file. Name node will forward request information to cloud storage static replica creation module. Cloud storage static replica creation modules select the information for creating the file such as nodes according to some kind of static strategy and back to the cloud-client. The namenode will record the access information when cloud-client access the data. Cloud storage dynamic replica creation module will get visit history from namenode and create a replica.

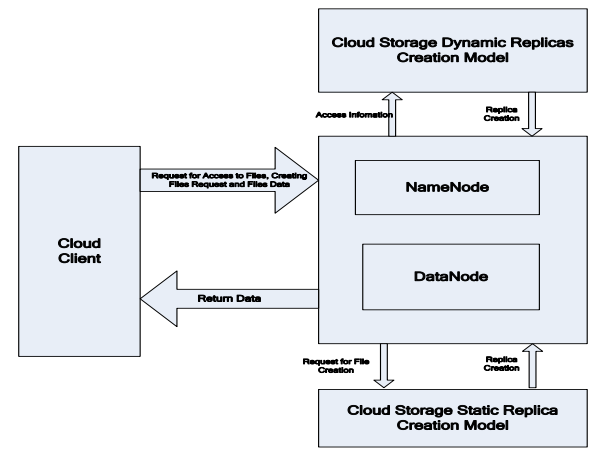

Figure1 Cloud storage to create a copy of the model

\section{DYNAMIC REPLICA CREATION STRATEGY}

Dynamic replica creation strategy utilizes the concept of a physical quantity, the acceleration response is the nature of the speed trend to predict file access frequency variation trend in order to identify hot spot data in next period and find the best node to create a replica.

\section{A. The Judgment of hot spot data}

Definition 1 Access speed: unit time the number of a file is accessed and this file is the speed of the formula is as follows:

$$
v=\frac{\sum_{t 2}-\sum_{t 1}}{t 2-t 1}
$$

Definition 2 access acceleration: Per unit time of a file access speed variation. A file in a certain period of time acceleration formula is as follows: 


$$
a_{t 2}=\frac{v_{t 2}-v_{t 1}}{\Delta t}
$$

$\mathrm{t} 1$ and $\mathrm{t} 2$ mean two moments, $\mathrm{t} 2>\mathrm{t} 1$. V1 and $\mathrm{v} 2$ mean the speed of file at $\mathrm{t} 1$ and $\mathrm{t} 2$. a means the access acceleration the theory of replica management.

The number of file replica can meet the current needs of the system. This theory is clearly established. Because the number of replica in this period is made by the system in the previous stage which can meet the system requirements.

It is very important for the replica management. Dynamic replica management strategy could focus on the prediction of the next period of time without need to concern the situation of the current file replica.

\section{B. The judgment algorithm of hot spot data}

We can get the hot spot data in the next period of time according to file the meaning of the acceleration, the acceleration of computation, numerical comparison. The speed of file response to the frequency of the file to be accessed which usually refers to a certain period of time. The acceleration of file response to the trend of speed and determine whether it is hot spot data. Given a threshold value $\mathrm{H}$, it can be identified as hot spot data when $\mathrm{a}>\mathrm{H}$. Specific steps are as follows:

1) Time Bucket t;

2) calculate the visits of file within t;

3) calculate the acceleration of each file within t;

4) Traversing the acceleration of all files, $i$ is a hot spot data when $\mathrm{a}>\mathrm{h}$ and back to the beginning;

5) The program jumps to the first step.

\section{Node selection of hot spot data}

The node selection of hot spot data is to select the best node for the hot spot data to store data.

When the node selection is not reasonable, the new created replica cannot play a good role, and the node load imbalance even collapse phenomenon appear easily. This is a huge burden on the system.

When a node has a very high frequency of access to a file in the current period in the system, it is concluded that the node is the main cause of the data become hot spots data. According to the continuity of access, it can be concluded that the node becomes hotspot node with high possibility in the next time, so we select this node as a hotspot node. In the process of concrete operation, Iterate through all the clients which access all of the hot spots data, we can select the node which has the highest frequency of access of all the clients as the hot node.

\section{V . EXPERIMENT SIMULATION AND RESULT ANALYSIS}

\section{A. Experiment simulation}

Replication strategy in the cloud storage environment simulation is difficult to achieve, in this paper, using the homemade simulation system simulation for simulation. By comparing this paper's replication strategy to other replication strategy, former has better performance in the experiment. A simple description about the experiment before analysis of the results, this experiment is a simulation system to simulate a real cloud storage environment, but because of cloud storage is large and complex system, so in order to make experiment more simple, the result more accurate, ignored during the experiment that has nothing to do with those factors of replication strategy. The following assumption:

1) Name node has a good performance, fast enough to support the whole system running, and does not fail in operation.

2) The performance of all data nodes are excellent, and can be guaranteed not to break down during the experiment. All the data node has the same storage space.

3) All switches performance optimal well enough to ensure the smooth when the network transmission. The switching time of all switches is equal.

Fig. 2 shows the network topology structure adopted in the experiment in this paper, the circle means server, and data node numbered from $0 \sim 9$ and numeral $\mathrm{N}$ is the server name node. Square in the figure means the switch which Numbered from $0 \sim 5$. Figure in the line said cables, switches, servers, connected via Ethernet cable, shown in the figure is the connection between the server and switches.

This experiment concerns that how to choose the hot spot data and how to choose a node to store the replica and what is the output results about replica creation tragedy.

For experimental purposes, two algorithms in this article are running results and analyze the results through process simulation.

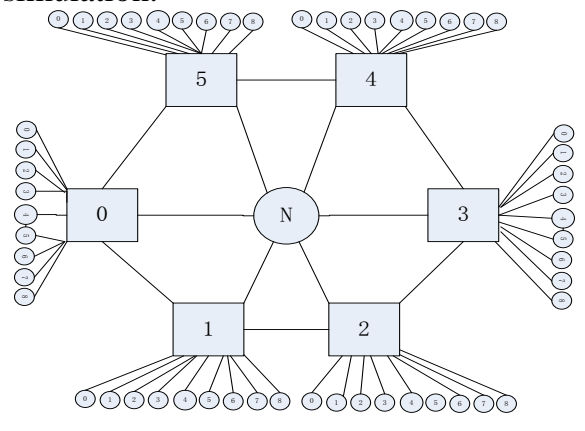

Figure 2 Network topology

\section{B. The analysis of experimental results}

As is shown in figure 5.3, in initial phase, the network transmission time increases with the increase of network access frequency and acceleration replica strategy is triggered with the increase of the frequency, then the growth of network transmission time is obviously decreased. This means acceleration strategy can predict the data whose access frequency is increased quickly to increase the replica. Figure 5 is a chart about acceleration replica management strategy compared with the best client replica management, the ordinate represents the overall network transmission time of the best client replica strategy minus the overall network delay of acceleration replica strategy and the horizontal represents the number of experiments. Two files are set in this experiment, and in one file the change of network demand is not big, in another file the growth of network demand is more obvious with the increase of the number of experiments. Two creating strategies create the same number of copies 
during the process of operation, as shown in 4. It describes the network transmission time difference of two strategies created by two policies changes along with network traffic increasing trend in figure 4 cases. As can be seen from the figure, replication strategy in acceleration way has obvious effect in reducing the network delay, and increased by file of the network demands. This shows, the replication created by acceleration replication strategy has a higher efficiency in reducing the network latency, very suitable for finding increasing demand network file demands, thus creating a replication for it and very suitable for the characteristics that cloud storage can storage mass files.

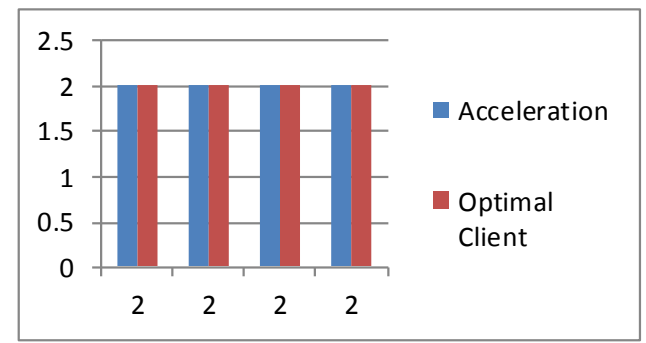

Figure 3 Acceleration strategy with frequency variation

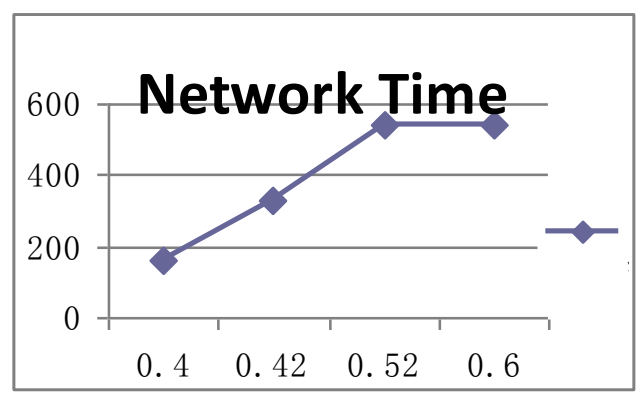

Figure 4 The number of copies

\section{CONCLUSION}

In this paper, we introduced the research status of replica create strategy, and the cloud storage dynamic replica creation module is put forward, and the replica creation module based on acceleration is put forward reference to physical concept acceleration strategy. Finally, through the simulation experiments, the replica creation strategy and the best client replica strategy are simulated under the same condition of simulation, we can conclude that the replica creation strategy based on acceleration has better effects on reducing network latency, which shows that it can predict the next hot data more accurately, and the cloud storage is also improved on the performance.

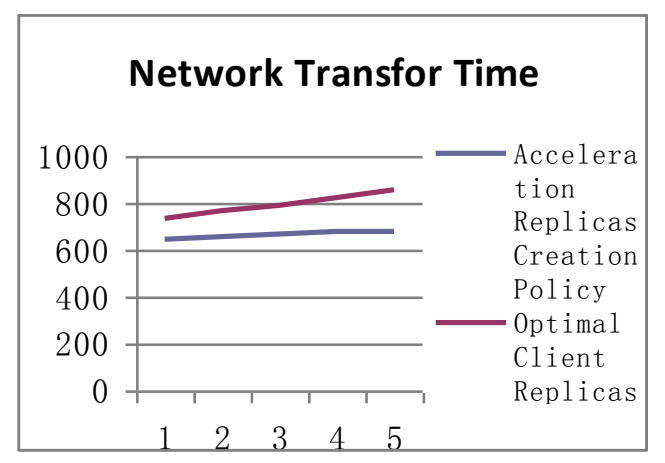

Figure5 Network transmission time ratio

\section{ACKNOWLEDGMENT}

This work is supported by the National Natural Science Foundation of China under Grant No. 71161007, the Social Science Fund Project of Ministry of Education under Grant No. 10YJCZH049, the Natural Science Foundation of Hainan under Grant No. 612132, the Key Science and Technology Project of Hainan under Grant No. ZDXM20120061 and ZDXM20130078.

\section{REFERENCE}

[1] Bonvin N, Papaioannou T G, Aberer K. Dynamic cost-efficient replication in data clouds $[\mathrm{C}] / / \mathrm{ACDC} 09$. Barcelona, Spain, 2009.

[2] Pinheiro E, Weber W D, Barroso L A. Failure trends in a large disk drive population [C] // Proc of 5th USENIX Conference on File and Storage Technologies ( FAST'07) . SanJose, CA, USA, 2007.

[3] Sun White paper: Cloud Computing Architecture [D] . 2009: 6.

[4] Apache Hadoop. http://hadoop.apche.org/ ,2012

[5] Xianglong Ye; Mengxing Huang; Donghai Zhu; Peng Xu; , "A Novel Blocks Placement Strategy for Hadoop," Computer and Information Science (ICIS), 2012 IEEE/ACIS 11th International Conference on , vol., no., pp.3-7, May 30 2012-June 12012

[6] RANGANATHAN K, FOSTER I. Design and evaluation of dynamic replication strategies for a high2performance data grid[C] //Proc of International Conference on Computing in High Energy and Nuclear Physics. Beijing: [s. n. ], 2001.

[7] Cameron DG, Carvjal-Schiaffino R. Evaluating scheduling and replica optimization strategies in OptorSim. In: Proc. of the 4th Int'l Workshop on Grid Computing (Grid 2003). Phenix: IEEE CS Press, 2003.

[8] Hassan and Ramaswamy. Message Replication in Unstructured Peer-To-Peer Network[C]. In Proc. International Conference on Networking Applications and Worksharing,. Nov 2007:pp.337-344. 2007

[9] Feng guofu, Zhang jincheng, Gu qing, Lu sanglu, Chen daoxu. A unstructured P2P overlay network topology based active replication strategy [J]. Journal of Software, 9(18):pp.2226-2234. 2007

[10] Kyungbaek Kim. Time-Related Replication for P2P Storage System[C]. In Proc. Seventh International Conference on Netwrking .April 2008:pp.351-356. 2008

[11] Xin Sun, Jun Zheng, Qiongxin Liu, Yushu Liu. Dynamic Data Replication Based on Access Cost in Distributed Systems[C]. In Proc. $4^{\text {th }}$ International Conference on Computing Sciences and Convergence Information Technology. pp.829-837. 2009.

[12] Xu jing, the research of replica stragedy in cloud storage [D], Hefei, University of science and technology of China, 2011. 